\title{
Dark matter as a solution to muonic puzzles
}

\author{
Maxim Perelstein and Yik Chuen San \\ Laboratory for Elementary Particle Physics, Cornell University, Ithaca, New York 14853, USA
}

(Received 21 November 2020; accepted 9 February 2021; published 25 February 2021)

\begin{abstract}
We propose a simple model in which dark matter particle exchanges mediate a new quantum force between muons and nucleons, resolving the proton charge radius puzzle. At the same time, the discrepancy between the measured anomalous magnetic moment of the muon and the Standard Model prediction can be accommodated, and thermal relic abundance of the dark matter candidate is consistent with observations. The dark matter particle mass is in the $\mathrm{MeV}$ range. We show that the model is consistent with a variety of experimental and observational constraints.
\end{abstract}

DOI: 10.1103/PhysRevD.103.035032

\section{INTRODUCTION}

Observational evidence for the existence of dark matter (DM) is overwhelming. While DM comprises most of the matter in today's Universe, and contributes about $20 \%$ of the total energy density, there is no known elementary particle that can account for it. Many candidate theories have been proposed, extending the Standard Model (SM) of particle physics to include one or more dark matter particles. In many theories, DM particles have potential experimental or observational signatures going beyond the purely gravitational effects that have been observed. However, no nongravitational signature of DM has been conclusively established so far.

In this paper, we propose that dark matter particles are directly responsible for explaining a long-standing puzzle in particle physics, the proton charge radius anomaly. The value of the proton charge radius measured using Lamb shift in muonic hydrogen $[1,2]$ does not agree, at about $5 \sigma$ level, with the value obtained from electron-proton scattering and electron hydrogen spectroscopy [3,4]. A similar discrepancy was observed in muonic deuterium [5]. We note that some recent data on $e p$ scattering [6] and $\mathrm{H}$ spectroscopy $[7,8]$ are in better agreement with muonic hydrogen results, while others [9] confirm the discrepancy. At present, the sources of disagreement among electronic experiments are not understood, and in this paper, we take the point of view that the anomaly is real and demands an explanation in terms of Beyond-the-Standard Model (BSM) phenomena. (For previous works that proposed BSM explanations of the proton charge radius puzzle, see, e.g., [10-14].)

Published by the American Physical Society under the terms of the Creative Commons Attribution 4.0 International license. Further distribution of this work must maintain attribution to the author(s) and the published article's title, journal citation, and DOI. Funded by SCOAP ${ }^{3}$.
The key ingredient of our proposal is the idea that loop diagrams involving light dark matter states can induce a new "quantum" force between SM particles $[15,16]$. Since the flavor structure of DM couplings to the SM is unconstrained, it is plausible that this new force may be felt by muons but not electrons. As will be shown below, such flavor-dependent quantum force can account for the proton charge radius puzzle, without conflict with any existing experimental constraints. At the same time, the dark matter particle responsible for this force can be a thermal relic consistent with the measured cosmological DM abundance, as well as with all known bounds on DM properties. The DM particle is predicted to have a mass of about $10 \mathrm{MeV}$, an interesting range from the point of view of direct detection experiments.

Another prominent experimental anomaly involving the muon is the anomalous magnetic moment $a_{\mu}$, whose measured value differs from the SM prediction by about $3 \sigma$ [17-19]. While nonperturbative SM contributions may account for some or all of this discrepancy, in this paper, we take the point of view that it is real and requires a BSM explanation. It turns out that in our model, the effect of DM loops on $a_{\mu}$ is subdominant to the shift induced by the mediator particles which connect DM and SM sectors. We show that this shift can indeed account for the observed discrepancy, and demonstrate a set of parameters for which $a_{\mu}$ and proton charge puzzles are simultaneously solved, DM particle is a thermal relic with correct relic density, and all experimental and observational constraints are satisfied. It is remarkable that the simple model presented here can account for such a diverse set of data pointing to physics beyond the SM.

\section{MODEL}

We introduce a DM field $\chi$, a Dirac fermion with no SM gauge charges. DM is coupled to the SM via two real-scalar 


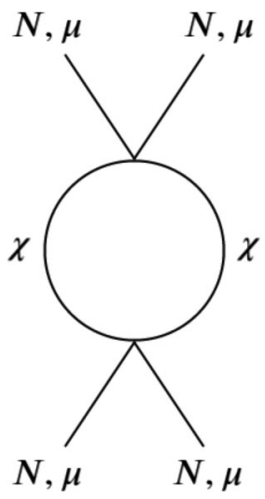

FIG. 1. One-loop diagram involving exchange of dark matter particle $\chi$ that induces a new force between muons and protons.

mediator fields, a leptophilic mediator $X$, and a leptophobic mediator $X^{\prime}$. Both mediators are also SM gauge singlets. At energies below the QCD confinement scale, where all physics relevant for this study takes place, the interaction Lagrangian is given by

$\mathcal{L}_{\text {int }}=-g \bar{\mu} \mu X-\left(g_{p}^{\prime} \bar{p} p+g_{n}^{\prime} \bar{n} n\right) X^{\prime}-y \bar{\chi} \chi X-y^{\prime} \bar{\chi} \chi X^{\prime}$,

where $\mu, p$, and $n$ are muon, proton, and neutron fields, respectively. Throughout this paper, we will study the regime in which both mediators are much heavier than the DM particle, $m_{X}, m_{X^{\prime}} \gg m_{\chi}$, and can be integrated out, leading to an effective Lagrangian

$$
\mathcal{L}_{\text {eff }}=-\frac{y g}{m_{X}^{2}} \bar{\chi} \chi \bar{\mu} \mu-\frac{y^{\prime} g_{p}^{\prime}}{m_{X^{\prime}}^{2}} \bar{\chi} \chi \bar{p} p-\frac{y^{\prime} g_{n}^{\prime}}{m_{X^{\prime}}^{2}} \bar{\chi} \chi \bar{n} n+\ldots
$$

The quantum force between proton and muon, arising from the diagrams in Fig. 1, provides a new contribution to the Lamb shift in muonic hydrogen, resolving the proton charge radius puzzle.

A few comments are in order. For simplicity, we assumed that the mediator $X$ (and therefore the DM) couples to muon but not to the electron. While the flavor-dependent nature of this coupling is crucial to resolving the proton charge radius puzzle, a nonzero value of electron coupling (e.g., a plausible scenario in which $g_{\ell} \propto m_{\ell}$ ) can be introduced without altering the basic picture. Further, $X^{\prime}$ is generically expected to couple to pions and other mesons. We do not include such couplings since they would play no role in our analysis. Finally, while the interactions in Eq. (1) are sufficient to explain the proton charge radius and the $a_{\mu}$ anomaly, requiring that $\chi$ be a thermal relic with observed cosmological abundance necessitates an additional interaction involving neutrinos $\nu$,

$$
\Delta \mathcal{L}_{\text {int }}=-\lambda \bar{\nu} \nu X
$$

if neutrinos are Dirac, or its Majorana counterpart. This interaction can arise from the operator $X(H L)^{2}$ above the weak scale, and its strength is a priori unrelated to the coupling of $X$ to charged muons which arises from the operator $X(H L) \mu_{R}$.

We also note that if $X$ and $X^{\prime}$ were replaced with a single mediator, coupled to both muons and quarks, the dominant new physics effect in muonic hydrogen would come from a tree-level exchange of the mediator, rather than the DMinduced quantum force. To leading order, this model would in fact be identical to that already considered in [14].

\section{RESULTS}

Using the above model, we performed a fit to relevant experimental data and observational constraints on DM properties. The results of the fit are summarized in Figs. 2-4. The model can explain the proton charge radius puzzle, the $a_{\mu}$ anomaly, and the observed DM relic density, while maintaining consistency with all known experimental and observational constraints. A sample benchmark point in the model parameter space which satisfies these requirements is shown in Table I. Some details of the analysis are presented below.

\section{A. Proton charge radius}

The fact that the proton has a finite size (with radius $r_{p}$ ) introduces shifts in energy levels of hydrogenlike atoms [1]. In particular, there would be a change in the energy difference between the $2 S$ and $2 P$ levels, i.e., Lamb shift $\Delta E_{\mathrm{Lamb}}$. By measuring $\Delta E_{\mathrm{Lamb}}$, one is able to deduce the value of $r_{p}$. In our model, the extra non-SM contribution to the Lamb shift in muonic hydrogen arises at one loop from the diagram in Fig. 1. In the nonrelativistic limit, this interaction can be captured by a potential between protons and muons [16],

$$
V(r)=-\frac{3}{4 \pi^{3} r}\left(\frac{y g}{m_{X}^{2}}\right)\left(\frac{y^{\prime} g_{p}^{\prime}}{m_{X^{\prime}}^{2}}\right) \frac{m_{\chi}^{2}}{r^{2}} K_{2}\left(2 m_{\chi} r\right),
$$

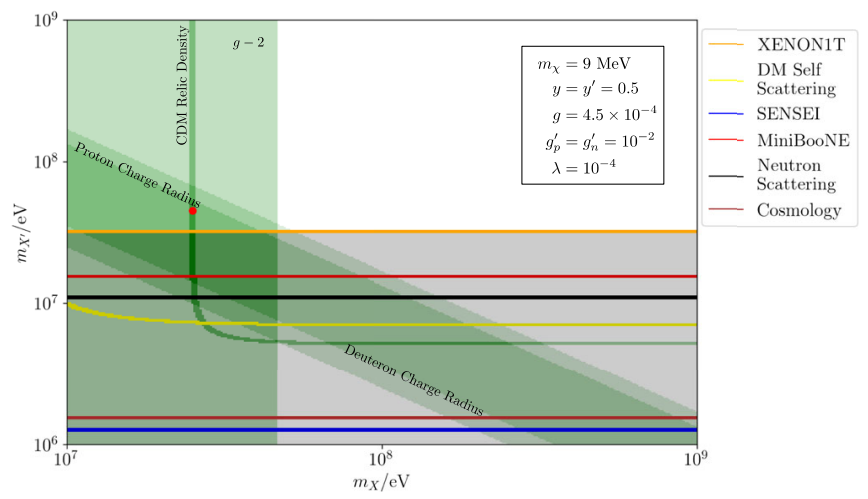

FIG. 2. Fit to experimental data indicating non-SM physics (green) in the plane of mediator particle masses, $m_{X}$ and $m_{X^{\prime}}$, with the other parameters fixed to the values listed in Table I. Relevant experimental and observational constraints on DM and mediator particles are also shown; the shaded areas are ruled out. 


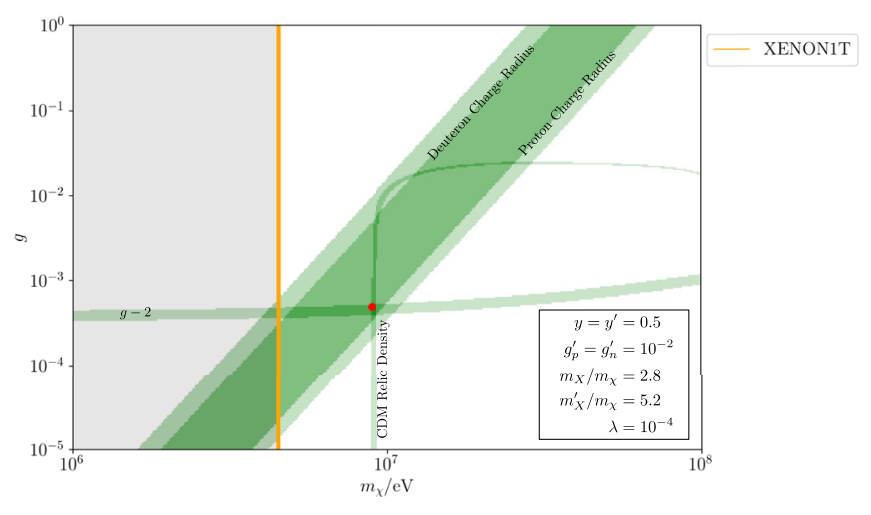

FIG. 3. Fit to experimental data indicating non-SM physics in the plane of DM particle mass $m_{\chi}$ and the leptophilic mediator coupling to muons, $g$, with the other parameters fixed to the values listed in Table I.

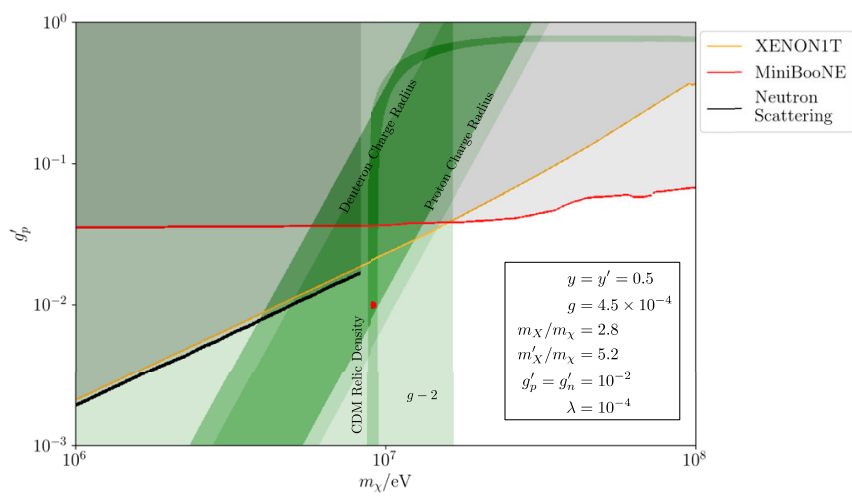

FIG. 4. Fit to experimental data indicating non-SM physics in the plane of DM particle mass $m_{\chi}$ and the leptophobic mediator coupling to protons, $g_{p}^{\prime}$, with the other parameters fixed to the values listed in Table I. Relevant experimental and observational constraints on DM and mediator particles are also shown.

where $K_{2}$ is the modified Bessel function of the second kind of order 2. As a result, there is a new contribution to $\Delta E_{\text {Lamb }}$ in muonic hydrogen, given by

$$
\begin{aligned}
\Delta E_{\mathrm{L}} & =\langle 2 S|V| 2 S\rangle-\langle 2 P|V| 2 P\rangle \\
& =-\frac{3}{8 \pi^{3}} \frac{y y^{\prime} g g_{p}^{\prime}}{m_{X}^{2} m_{X^{\prime}}^{2}} \frac{m_{\chi}^{2}}{a^{3}} \mathcal{J}\left(x_{0}, a\right) .
\end{aligned}
$$

Here

$$
\mathcal{J}\left(x_{0}, a\right)=\int_{x_{0}}^{\infty} \mathrm{d} x \frac{6(1-x)+x^{2}}{6 x} e^{-x} K_{2}\left(2 m_{\chi} a x\right),
$$

TABLE I. Model parameters at the benchmark point.

\begin{tabular}{lcccccccc}
\hline \hline$m_{\chi}$ & $m_{X}$ & $m_{X^{\prime}}$ & $g$ & $g_{p}^{\prime}$ & $g_{n}^{\prime}$ & $y$ & $y^{\prime}$ & $\lambda$ \\
\hline $9 \mathrm{MeV}$ & $25 \mathrm{MeV}$ & $45 \mathrm{MeV}$ & $4.5 \times 10^{-4}$ & 0.01 & 0.01 & 0.5 & 0.5 & $10^{-4}$ \\
\hline \hline
\end{tabular}

$a$ is the Bohr radius of muonic hydrogen, and $x_{0} \approx$ $\left(a \Lambda_{\mathrm{QCD}}\right)^{-1}$ is the short-distance cutoff corresponding to the breakdown of the effective field theory description in Eq. (1) at length scales below $\mathcal{O}\left(\Lambda_{\mathrm{QCD}}\right)$. We do not include the additional contribution to the Lamb shift from length scales below $r_{0}$, which can only be calculated within a specific UV completion of Eq. (1). To estimate the associated theoretical uncertainty, we vary the value of the cutoff by a factor of 2 around the assumed central value $\left(a \Lambda_{\mathrm{QCD}}\right)^{-1}$. This uncertainty is reflected in the width of the "proton charge radius" band in Figs. 2-4. Our fit assumes that this effect fully accounts for the "extra" Lamb shift measured in the muonic hydrogen, compared to the baseline value inferred from electronic hydrogen and $e p$ scattering: $\Delta E_{\mathrm{L}}=-0.307(56) \mathrm{meV}$. This is appropriate since in our model the electron does not couple to DM and hence is unaffected by the new force.

\section{B. Muonic deuterium}

After the proton charge radius puzzle was discovered, there has been interest in performing similar experiments with other muonic atoms, in particular muonic deuterium $\mu D$. It was reported that the deuteron charge radius $r_{d}$ extracted from $\mu D$ shows similar discrepancy from worldaveraged CODATA 2014 value [5,4]. When comparing against spectroscopic values of $r_{d}$ that involve deuterium only (i.e., independent of $r_{p}$ ), this discrepancy is reduced to $3.6 \sigma$ [20], but is still statistically significant. We therefore require that our model produces an extra contribution to Lamb shift in $\mu D$ [5]: $\Delta E_{\mathrm{L}}^{\mu D}=-0.438(59) \mathrm{meV}$. The Lamb shift in $\mu D$ is due to dark matter-mediated quantum force and is given by

$$
\Delta E_{\mathrm{L}}^{\mu D}=-\frac{3}{8 \pi^{3}} \frac{y g}{m_{X}^{2}} \frac{y^{\prime}\left(g_{p}^{\prime}+g_{n}^{\prime}\right)}{m_{X^{\prime}}^{2}} \frac{m_{\chi}^{2}}{a_{\mu D}^{3}} \mathcal{J}\left(x_{0 D}, a_{\mu D}\right),
$$

where $a_{\mu D}$ is the Bohr radius of the muonic deuterium system, $r_{D}$ is the deuteron radius, and $x_{0 D}=r_{D} / a_{\mu D}$. The shift depends on both $g_{n}^{\prime}$ and $g_{p}^{\prime}$, while various combinations of these couplings are constrained by nuclear physics experiments (see below). As shown in Fig. 5, isospinpreserving coupling $g_{p}^{\prime}=g_{n}^{\prime}$ is consistent with both the deuteron charge radius and nuclear physics constraints.

\section{Muon anomalous magnetic moment}

The leading new contribution to $a_{\mu}$ is given by the oneloop diagram shown in Fig. 6. Note that this contribution is independent of the dark matter candidate $\chi$ itself, which only enters at the two-loop level. The shift in $a_{\mu}$ is given by

$$
\Delta a_{\mu}=\frac{2 g^{2}}{(4 \pi)^{2}} \int_{0}^{1} \mathrm{~d} x \frac{(1-x)^{2}(1+x)}{(1-x)^{2}+x\left(m_{X} / m_{\mu}\right)^{2}} .
$$




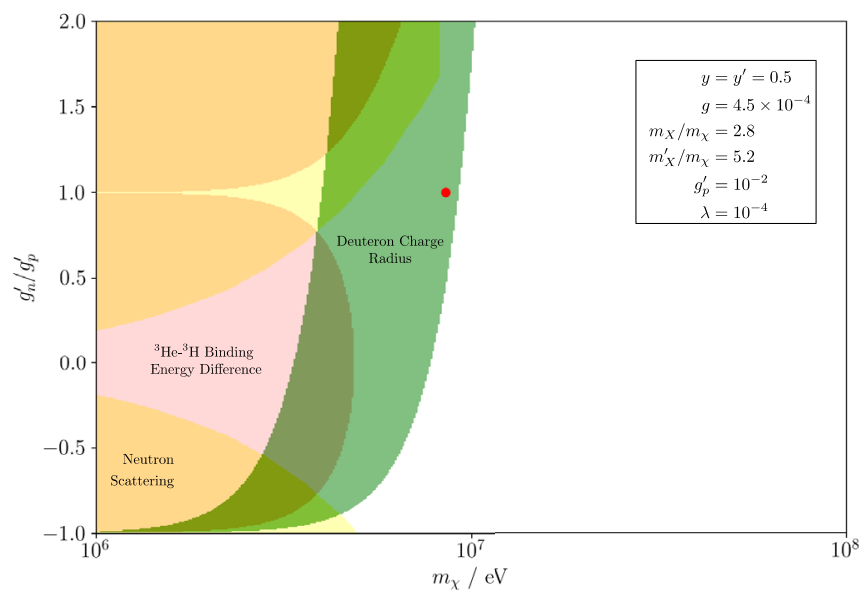

FIG. 5. Fit to muonic deuterium data, as well as constraints from nuclear physics experiments, in the plan of the mediator coupling ratio $g_{n}^{\prime} / g_{p}^{\prime}$ and the DM particle mass $m_{\chi}$. The other parameters are fixed to the values listed in Table I.

In our fit, we assume that this effect fully accounts for the experimental discrepancy $\Delta a_{\mu}=287(80) \times 10^{-11}$, within 2 standard deviations.

\section{Relic density}

We assume that $\chi$ is a thermal relic and that it accounts for all of the observed cosmological DM abundance, $\Omega h^{2}=0.120 \pm 0.001$ [21]. With interactions in Eq. (1) and $m_{\chi} \sim \mathcal{O}(\mathrm{MeV})$, the dominant DM annihilation channel at the time of freeze-out is $\chi \chi \rightarrow 2 \gamma$; see Fig. 7. The leading ( $p$-wave) contribution to the cross section in the nonrelativistic limit is given by

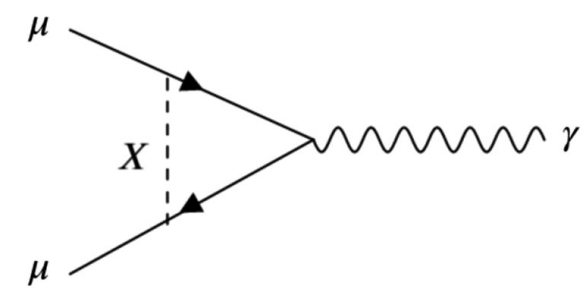

FIG. 6. Contribution to muon anomalous magnetic moment due to the mediator particle $X$.

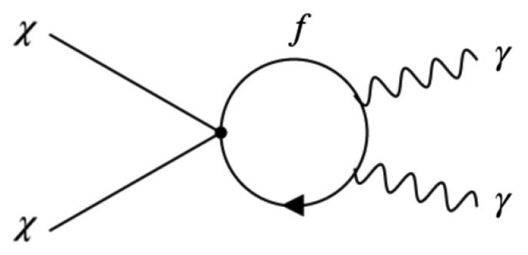

FIG. 7. Dominant annihilation channel for $\chi$ at the time of freeze-out, if coupling to neutrinos are absent.

$$
\sigma_{0}=\frac{3 e^{2}}{32 \pi^{3}}\left|m_{\mu} I\left(\tau_{\mu}\right) \frac{y g}{m_{X}^{2}}+m_{p} I\left(\tau_{p}\right) \frac{y^{\prime} g_{p}^{\prime}}{m_{X^{\prime}}^{2}}\right|^{2} .
$$

Here the loop function $I\left(\tau_{f}\right)$ for a fermion $f$ is defined by

$$
I\left(\tau_{f}\right)=\int_{0}^{1} \mathrm{~d} x \int_{0}^{1-x} \mathrm{~d} y \frac{1-4 x y}{\tau_{f}-x y},
$$

with $\tau_{f}=m_{f}^{2} / s$, where $\sqrt{s}$ is the center-of-mass energy of the scattering process. $\sigma_{0}$ can be used to compute the relic density of $\chi$ [22] by solving the Boltzmann equation numerically.

We find that the $\chi \chi \rightarrow 2 \gamma$ cross section is too small to provide the observed relic density for model parameters required to fit the proton charge radius and $a_{\mu}$ anomalies. A simple solution is to consider an additional annihilation channel, $\chi \chi \rightarrow \nu \nu$, via the interaction in Eq. (3). The cross section is given by $\sigma_{0}=\frac{3}{4 \pi}\left(\frac{y \lambda}{m_{X}^{2}}\right)^{2} m_{\chi}^{2}$. Since this final state arises at tree level, it naturally dominates over the $2 \gamma$ channel. With this addition, all three constraints can be satisfied simultaneously; see Figs. 2-4.

In addition to fits to the data indicating deviations from the SM, a number of constraints from data and observations consistent with the SM have to be taken into account.

\section{Dark matter self-scattering}

Tree-level exchanges of mediator particles $X$ and $X^{\prime}$ induce DM short-range self-interactions of the form

$$
\mathcal{L}_{\text {self }}=-\left(\frac{y^{2}}{m_{X}^{2}}+\frac{y^{\prime 2}}{m_{X^{\prime}}^{2}}\right)(\bar{\chi} \chi)^{2} .
$$

DM self-scattering cross sections at low velocities are bounded by observations of halo shapes [23,24]: $\sigma_{T} / m_{\chi} \lesssim 1 \mathrm{~cm}^{2} / \mathrm{g}$, where $\sigma_{T}$ is the momentum-transfer cross section defined by

$$
\sigma_{T} \equiv \int \mathrm{d} \Omega \frac{\mathrm{d} \sigma_{\chi \chi \rightarrow \chi \chi}}{\mathrm{d} \Omega}(1-\cos \theta) .
$$

This constraint translates into an upper bound on the DM mass

$$
m_{\chi} \lesssim\left(\frac{y^{2}}{m_{X}^{2}}+\frac{y^{\prime 2}}{m_{X^{\prime}}^{2}}\right)^{-2} \times 1.16 \mathrm{MeV},
$$

where $m_{X^{\prime}}$ and $m_{X}$ have been normalized to units of $10 \mathrm{MeV}$.

\section{Dark matter direct detection}

Direct detection of dark matter in the $\mathrm{MeV}$ mass range has been the subject of much interest recently $[25,26]$. Most techniques rely on detection of DM scattering on electrons. 
In our model, this channel is not available, since by construction DM does not couple to electrons. However, scattering on a nucleon can occur, with cross section

$$
\sigma(\chi N \rightarrow \chi N)=\frac{1}{\pi}\left(\frac{g_{N}^{\prime} y^{\prime}}{m_{X^{\prime}}^{2}}\right)^{2}\left(\frac{m_{\chi} m_{N}}{m_{\chi}+m_{N}}\right)^{2},
$$

where $N=n$ or $p$. For our benchmark point, this cross section is about $6 \times 10^{-32} \mathrm{~cm}^{2}$. This is about half an order of magnitude below the strongest current constraint from nonobservation of signal due to energetic DM component generated through collisions with cosmic rays [27,28], shown by the XENON-1T curve in Fig. 4. MeV-scale dark matter can also be detected using the Migdal effect [29-33]. However, the recent results from SENSEI Collaboration [34] are not yet sensitive enough to constrain our model.

\section{Early Universe cosmology}

Measurements of cosmic microwave background (CMB) place strong constraints on possible reionization due to DM annihilations [35]. However, in our model, $\chi$ annihilation proceeds in $p$ wave and thus not subject to this constraint. CMB data together with the Lyman-alpha forest flux power spectrum from the Sloan Digital Sky Survey constrains the elastic scattering of DM on baryons [36,37]. This constraint is shown by the curve labeled "cosmology" on Fig. 2. In addition, a scenario where DM freeze-out occurs after neutrinos decouple from the rest of the SM plasma is constrained by the CMB bound on $\Delta N_{\text {eff }}$, since in this case the neutrino temperature at recombination would be raised relative to $T_{\gamma}$ by the entropy transferred from the DM. ${ }^{1}$ This argument imposes a lower bound $m_{\chi} \gtrsim$ a few $\mathrm{MeV}$ [38,39]. A similar bound is imposed by the success of big bang nucleosynthesis (BBN) [40-42]. Mediators $X$ and $X^{\prime}$ decouple from the thermal bath before the onset of the BBN, and the few mediator particles remaining after decoupling decay very quickly. Thus, the mediators play no role in the cosmological constraints.

\section{Dark matter mediator searches}

In addition to direct searches for dark matter particles, there are many experiments looking for mediator particles produced at colliders or in a fixed-target setup [25,26]. While most analyses present the results in terms of bounds on dark photons, which couple to both leptons and quarks proportional to their electric charges, the interpretation of interest to us is in terms of leptophilic or leptophobic mediators. Leptophilic mediator searches rely on their production via their interaction with electrons, making them insensitive to our model. (An exception is the recently

\footnotetext{
${ }^{1}$ We are grateful to Gordan Krnjaic for bringing this constraint to our attention.
}

reported NA64 search for scalars produced through their coupling to photons [43]. However, this search does not place relevant bounds on our model, since mediator couplings to photons are loop suppressed.) Thus, we only consider bounds from leptophobic mediator searches. With sub-MeV dark matter, the most stringent bound currently comes from the MINIBOONE experiment [26,44], which places a bound on the parameter $Y$ related to dark matter annihilation cross section (see referenced papers for the precise definition). In our model, this parameter is given by

$$
Y=\left(\frac{g_{p}^{\prime}}{e}\right)^{2} \frac{g_{p}^{\prime}}{4 \pi}\left(\frac{m_{\chi}}{m_{X^{\prime}}}\right)^{2} .
$$

Note that the mediator $X^{\prime}$ in our model is a scalar, while the MINIBOONE bounds were derived using a spin-1 mediator. An order-one correction to the bound may arise due to the differing kinematic acceptances and spin factors in the two cases, but we do not expect it to affect our conclusions.

\section{Nuclear interactions}

Leading non-SM contributions to nucleon-nucleon potential are given by

$$
\begin{aligned}
V_{N_{1} N_{2}}= & -\frac{g_{N_{1}}^{\prime} g_{N_{2}}^{\prime}}{4 \pi r} e^{-m_{X^{\prime}} r} \\
& -\frac{3}{4 \pi^{3}}\left(\frac{y^{\prime}}{m_{X^{\prime}}^{2}}\right)^{2} g_{N_{1}}^{\prime} g_{N_{2}}^{\prime} \frac{m_{\chi}^{2}}{r^{3}} K_{2}\left(2 m_{\chi} r\right) .
\end{aligned}
$$

This extra potential can be probed at various nuclear physics experiments. It turns out that the second term in $V_{N_{1} N_{2}}$, arising from the DM-loop exchange, is the most relevant for our analysis, since $1 / r_{\exp } \ll m_{\chi} \ll m_{X^{\prime}}$, where $r_{\text {exp }}$ is the length scale probed by the experiments. The relevant constraints are summarized in Fig. 5.

The binding energy difference between ${ }^{3} \mathrm{He}$ and ${ }^{3} \mathrm{H}$ has been well stablished to be caused by Coulomb force and charge asymmetry of nuclear forces [45-47]. In order not to spoil this agreement, the non-SM contribution is required to be less than $30 \mathrm{keV}$ [14]. It is worth noting that this contribution is proportional to $\left(g_{p}^{\prime 2}-g_{n}^{\prime 2}\right)$ and vanishes in the isospin limit; see Fig. 5.

The charge-independence breaking scattering length is defined as

$$
\Delta a=\frac{1}{2}\left(a_{n n}+a_{p p}-2 a_{n p}\right)
$$

where $a_{N_{1} N_{2}}$ is the scattering length between two nucleons $N_{1}$ and $N_{2}$. Experimental and theoretical values for $\Delta a$ are known to be $5.64 \pm 0.60 \mathrm{fm}$ [48] and $5.6 \pm 0.5 \mathrm{fm}$ [49], respectively. Our model gives an extra contribution 


$$
\delta a^{\text {th }}=-\frac{3 m_{N}}{\pi^{2}}\left(\frac{y^{\prime}}{m_{X^{\prime}}}\right)^{2} m_{\chi}^{2}\left(g_{p}^{\prime}-g_{n}^{\prime}\right)^{2} \log \left(\frac{m_{\chi}^{2}}{\Lambda^{2}}\right),
$$

where $m_{N}$ is the nucleon mass and $\Lambda$ is the cutoff scale of the effective theory. We require $\delta a^{\text {th }}<1.6 \mathrm{fm}$ to maintain agreement between experimental and theoretical values of $\Delta a$ at $2 \sigma$ level. This constraint again vanishes in the isospin limit.

Scattering lengths between cold neutrons and nuclei can be measured by different methods, such as Bragg diffraction and the transmission method [50]. In our model, this scattering length is given by Eq. (18) by replacing $\left(g_{p}^{\prime}-g_{n}^{\prime}\right)^{2}$ with $g_{n}^{\prime 2}$ or $g_{n}^{\prime} g_{p}^{\prime}$. By comparing the scattering lengths measured by different methods, bounds can be placed on contributions from noncontact operators. The detailed analysis can be found in [51]. Neutron scattering places the most restrictive nuclear-physics bound on the model in the isospin-symmetric limit [16].

\section{Meson decays}

Our model contains a light mediator $X^{\prime}$, which couples to quarks and decays mainly invisibly, with a subdominant loop decay to $2 \gamma$. This can affect meson decays. Parity forbids $X^{\prime}$ mixing with the pion, so that $\pi \rightarrow$ inv. and $\pi \rightarrow 2 \gamma$ decay rates are not affected. Charge conjugation symmetry forbids the decay $\pi^{0} \rightarrow X^{\prime} \gamma$, so unlike the dark photon, there is no constraint on our model from this decay. The strongest relevant bound comes from the decay $K \rightarrow \pi+X^{\prime}$, with subsequent invisible $X^{\prime}$ decay. Experimentally, this decay would appear as a new contribution to $K \rightarrow \pi+$ inv [52]. However, $X^{\prime}$ couplings to quarks are model dependent, which can strongly affect this constraint. In particular, if $X^{\prime}$ only couples to down-type quarks, there is no one-loop contribution to $K \rightarrow \pi X^{\prime}$ and our model is easily consistent with this bound. In the future, it will be interesting to construct ultraviolet completions of our model to explore possible textures in mediator coupling to quarks and to analyze meson decay constraints within those theories where the relevant rates can be calculated explicitly.

\section{CONCLUSIONS}

In this paper, we presented a simple model of $\mathrm{MeV}$-scale Dirac fermion dark matter $\chi$ coupled to nucleons and muons, but not electrons. The quantum force due to $\chi$ loops is responsible for resolving the proton (and deutron) charge radius puzzles, while a scalar particle introduced to mediate DM-muon interactions can account for the discrepancy between the SM prediction for the anomalous magnetic moment of the muon and the measured value. If an additional interaction between $\chi$ and neutrinos is postulated, the former can be a thermal relic responsible for all of the observed DM abundance. We verified the existence of a region in the parameter space of the model where all of the above features are obtained simultaneously, while all known experimental and observational constraints on light DM and mediators are satisfied.

New experimental data will soon be available that will test our model on various fronts. Numerous efforts, e.g., MUSE experiment [53], are under way that will hopefully clarify the status of the proton charge radius puzzle. Anomalous magnetic moment of the muon measurement will be improved by the Fermilab Muon $g-2$ experiment [54]. Experimental exploration of $\mathrm{MeV}$-scale dark matter and dark sectors is an active and expanding area of research $[25,26]$. While our model has some amount of freedom in parameter choices, the available parameter space is finite and it is likely that this idea will be tested conclusively by the upcoming experiments in the near future.

\section{ACKNOWLEDGMENTS}

We are grateful to Gordan Krnjaic and Philip Tanedo for useful comments on the first version of this paper. This work was supported by the U.S. National Science Foundation Grants No. PHY-1719877 and No. PHY2014071.
[1] R. Pohl et al., The size of the proton, Nature (London) 466 , 213 (2010).

[2] A. Antognini et al., Proton structure from the measurement of $2 S-2 P$ transition frequencies of muonic hydrogen, Science 339, 417 (2013).

[3] P. J. Mohr, B. N. Taylor, and D. B. Newell, CODATA recommended values of the fundamental physical constants: 2006, Rev. Mod. Phys. 80, 633 (2008).

[4] P. J. Mohr, D. B. Newell, and B. N. Taylor, CODATA recommended values of the fundamental physical constants: 2014, Rev. Mod. Phys. 88, 035009 (2016).
[5] R. Pohl et al. (CREMA Collaboration), Laser spectroscopy of muonic deuterium, Science 353, 669 (2016).

[6] W. Xiong et al., A small proton charge radius from an electron-proton scattering experiment, Nature (London) 575, 147 (2019).

[7] A. Beyer et al., The Rydberg constant and proton size from atomic hydrogen, Science 358, 79 (2017).

[8] N. Bezginov, T. Valdez, M. Horbatsch, A. Marsman, A. Vutha, and E. Hessels, A measurement of the atomic hydrogen Lamb shift and the proton charge radius, Science 365, 1007 (2019). 
[9] H. Fleurbaey, S. Galtier, S. Thomas, M. Bonnaud, L. Julien, F. Biraben, F. Nez, M. Abgrall, and J. Guena, New Measurement of the 1S-3S Transition Frequency of Hydrogen: Contribution to the Proton Charge Radius Puzzle, Phys. Rev. Lett. 120, 183001 (2018).

[10] D. Tucker-Smith and I. Yavin, Muonic hydrogen and MeV forces, Phys. Rev. D 83, 101702 (2011).

[11] B. Batell, D. McKeen, and M. Pospelov, New ParityViolating Muonic Forces and the Proton Charge Radius, Phys. Rev. Lett. 107, 011803 (2011).

[12] C. E. Carlson and B. C. Rislow, New physics and the proton radius problem, Phys. Rev. D 86, 035013 (2012).

[13] S. G. Karshenboim, D. McKeen, and M. Pospelov, Constraints on muon-specific dark forces, Phys. Rev. D 90, 073004 (2014); 90, 079905(A) (2014).

[14] Y.-S. Liu, D. McKeen, and G. A. Miller, Electrophobic Scalar Boson and Muonic Puzzles, Phys. Rev. Lett. 117, 101801 (2016).

[15] S. Fichet, Quantum Forces from Dark Matter and Where to Find Them, Phys. Rev. Lett. 120, 131801 (2018).

[16] P. Brax, S. Fichet, and G. Pignol, Bounding quantum dark forces, Phys. Rev. D 97, 115034 (2018).

[17] G. Bennett et al. (Muon g-2 Collaboration), Final report of the Muon E821 anomalous magnetic moment measurement at BNL, Phys. Rev. D 73, 072003 (2006).

[18] M. Davier, A. Hoecker, B. Malaescu, and Z. Zhang, Reevaluation of the hadronic contributions to the, Eur. Phys. J. C 71, 1515 (2011); Erratum, Eur. Phys. J. C 72, 1874 (2012).

[19] K. Hagiwara, R. Liao, A. D. Martin, D. Nomura, and T. Teubner, Re-evaluated using new precise data, J. Phys. G 38, 085003 (2011).

[20] R. Pohl, F. Nez, T. Udem, A. Antognini, A. Beyer, H. Fleurbaey, A. Grinin, T. W. Hansch, L. Julien, F. Kottmann et al., Deuteron charge radius and rydberg constant from spectroscopy data in atomic deuterium, Metrologia 54, L1 (2017).

[21] N. Aghanim et al. (Planck Collaboration), Planck 2018 results. VI. Cosmological parameters, Astron. Astrophys. 641, A6 (2020).

[22] E. W. Kolb and M.S. Turner, The early Universe, Front. Phys. 69, 1 (1990).

[23] A. Kusenko and L. J. Rosenberg, Snowmass-2013 cosmic frontier 3 (CF3) working group summary: Non-WIMP dark matter, arXiv:1310.8642.

[24] J. Zavala, M. Vogelsberger, and M. G. Walker, Constraining self-interacting dark matter with the Milky Way's dwarf spheroidals, Mon. Not. R. Astron. Soc. Lett. 431, L20 (2013).

[25] J. Alexander et al., Dark sectors 2016 workshop: Community report, arXiv:1608.08632.

[26] M. Battaglieri et al., in U.S. cosmic visions: New ideas in dark matter, arXiv:1707.04591.

[27] T. Bringmann and M. Pospelov, Novel Direct Detection Constraints on Light Dark Matter, Phys. Rev. Lett. 122, 171801 (2019).

[28] C. Cappiello and J. F. Beacom, Strong new limits on light dark matter from neutrino experiments, Phys. Rev. D 100, 103011 (2019).
[29] M. Ibe, W. Nakano, Y. Shoji, and K. Suzuki, Migdal effect in dark matter direct detection experiments, J. High Energy Phys. 03 (2018) 194.

[30] M. J. Dolan, F. Kahlhoefer, and C. McCabe, Directly Detecting Sub-GeV Dark Matter with Electrons from Nuclear Scattering, Phys. Rev. Lett. 121, 101801 (2018).

[31] N. F. Bell, J. B. Dent, J. L. Newstead, S. Sabharwal, and T. J. Weiler, Migdal effect and photon bremsstrahlung in effective field theories of dark matter direct detection and coherent elastic neutrino-nucleus scattering, Phys. Rev. D 101, 015012 (2020).

[32] D. Baxter, Y. Kahn, and G. Krnjaic, Electron ionization via dark matter-electron scattering and the Migdal effect, Phys. Rev. D 101, 076014 (2020).

[33] R. Essig, J. Pradler, M. Sholapurkar, and T.-T. Yu, Relation between the Migdal Effect and Dark Matter-Electron Scattering in Isolated Atoms and Semiconductors, Phys. Rev. Lett. 124, 021801 (2020).

[34] L. Barak, I. M. Bloch, M. Cababie, G. Cancelo, L. Chaplinsky, F. Chierchie, M. Crisler, A. Drlica-Wagner, R. Essig, J. Estrada et al., Sensei: Direct-Detection Results on Sub-Gev Dark Matter from a New Skipper-CCD, Phys. Rev. Lett. 125, 171802 (2020).

[35] T. R. Slatyer, Indirect dark matter signatures in the cosmic dark ages. I. Generalizing the bound on s-wave dark matter annihilation from Planck results, Phys. Rev. D 93, 023527 (2016).

[36] K. K. Boddy and V. Gluscevic, First cosmological constraint on the effective theory of dark matter-proton interactions, Phys. Rev. D 98, 083510 (2018).

[37] W. L. Xu, C. Dvorkin, and A. Chael, Probing sub-GeV dark matter-baryon scattering with cosmological observables, Phys. Rev. D 97, 103530 (2018).

[38] C. M. Ho and R. J. Scherrer, Limits on MeV dark matter from the effective number of neutrinos, Phys. Rev. D 87, 023505 (2013).

[39] C. Boehm, M. J. Dolan, and C. McCabe, Increasing Neff with particles in thermal equilibrium with neutrinos, J. Cosmol. Astropart. Phys. 12 (2012) 027.

[40] E. W. Kolb, M.S. Turner, and T. P. Walker, The Effect of Interacting Particles on Primordial Nucleosynthesis, Phys. Rev. D 34, 2197 (1986).

[41] M. Hufnagel, K. Schmidt-Hoberg, and S. Wild, BBN constraints on MeV-scale dark sectors. Part I. Sterile decays, J. Cosmol. Astropart. Phys. 02 (2018) 044.

[42] K. M. Nollett and G. Steigman, BBN and the CMB constrain neutrino coupled light WIMPs, Phys. Rev. D 91, 083505 (2015).

[43] D. Banerjee et al. (NA64 Collaboration), Search for Axionlike and Scalar Particles with the NA64 Experiment, Phys. Rev. Lett. 125, 081801 (2020).

[44] A. Aguilar-Arevalo et al. (MiniBooNE DM Collaboration), Dark matter search in nucleon, pion, and electron channels from a proton beam dump with MiniBooNE, Phys. Rev. D 98, 112004 (2018).

[45] J. L. Friar and B.F. Gibson, Coulomb energies in $s$-shell nuclei and hypernuclei, Phys. Rev. C 18, 908 (1978). 
[46] V. Koch and G. A. Miller, Six quark cluster effects and binding energy differences between mirror nuclei, Phys. Rev. C 31, 602 (1985).

[47] G. Miller, B. Nefkens, and I. Šlaus, Charge symmetry, quarks and mesons, Phys. Rep. 194, 1 (1990).

[48] T. Ericson and G. Miller, Charge dependence of nuclear forces, Phys. Lett. 132B, 32 (1983).

[49] R. Machleidt and I. Slaus, The nucleon-nucleon interaction, J. Phys. G 27, R69 (2001).

[50] L. Koester, H. Rauch, and E. Seymann, Neutron scattering lengths: A survey of experimental data and methods, At. Data Nucl. Data Tables 49, 65 (1991).
[51] V. Nesvizhevsky, G. Pignol, and K. Protasov, Neutron scattering and extra short range interactions, Phys. Rev. D 77, 034020 (2008).

[52] E. Cortina Gil et al. (NA62 Collaboration), First search for $K^{+} \rightarrow \pi^{+} \nu \bar{\nu}$ using the decay-in-flight technique, Phys. Lett. B 791, 156 (2019).

[53] R. Gilman et al. (MUSE Collaboration), Technical design report for the Paul Scherrer institute experiment R-12-01.1: Studying the proton "radius" puzzle with $\mu p$ elastic scattering, arXiv:1709.09753.

[54] J. Grange et al. (Muon g-2 Collaboration), Muon (g-2) technical design report, arXiv:1501.06858. 